TITLE:

\title{
Minimizing Periodic Orbits with Regularizable Collisions in the n- Body Problem
}

\author{
$\operatorname{AUTHOR}(\mathrm{S})$ :
}

Shibayama, Mitsuru

\section{CITATION:}

Shibayama, Mitsuru. Minimizing Periodic Orbits with Regularizable Collisions in the nBody Problem. Archive for Rational Mechanics and Analysis 2011, 199(3): 821-841

\section{ISSUE DATE:}

2011-03

URL:

http://hdl.handle.net/2433/139552

\section{RIGHT:}

The final publication is available at www.springerlink.com; この論文は 出版社版でありません。引用の際には出版社版をご確認ご利用くださ $\omega_{\circ}$; This is not the published version. Please cite only the published version. 


\title{
Minimizing Periodic Orbits with Regularizable Collisions in the $n$-Body Problem
}

\author{
Mitsuru Shibayama
}

\begin{abstract}
There are various subsytems with two degrees of freedom in the Newtonian $n$-body problem, such as the collinear three-body problem and the isosceles three-body problem. After we determine a normal form of the Lagrangians of these subsystems, we prove the existence of periodic solutions with regularizable collisions for these systems. Our result includes several examples, such as Schubart's orbit with or without equal masses among others.
\end{abstract}

\section{$1 \quad$ Introduction and Results}

This paper is concerned with the Newtonian $n$-body problem which is given by the following set of ODEs:

$$
\ddot{q}_{i}=-\sum_{j \neq i} m_{j} \frac{q_{i}-q_{j}}{\left\|q_{i}-q_{j}\right\|^{3}} \quad q_{1}, \cdots, q_{n} \in \mathbb{R}^{d}
$$

where $m_{j}>0$ and $d=1,2,3$.

As a recent remarkable progress, by using the variational method Chenciner and Montgomery [3] proved the existence of a new periodic solution of figureeight shape to the planar three-body problem. Since then, a number of periodic and quasi-periodic solutions have been found as minimizers of variational formulation of the $n$-body problem in various different settings. In particular, Ferrario and Terracini [4] introduced the rotating circle property and showed that a collisionless solution having a certain symmetry exists, provided the group action of the symmetry satisfies the rotating circle property.

We study the subsystems with two degrees of freedom, such as the collinear three-body problem and the rhomboidal four-body problem among others. We can not apply [4] to these systems since the symmetries are too strong to satisfy the rotating circle property. Our goal is to prove the existence of periodic orbits which alternately repeat two partial collisions and which are smooth under a regularization of the singularities.

Schubart's orbit is a well-known example. This is a periodic solution in the collinear three-body problem with two equal masses where the particle between two with the equal masses repeats binary collisions with others alternately. 
Schubart [8] numerically found this solution, and Moeckel [5] and Venturelli [11] rigorously proved the existence by using the topological and variational methods respectively. The existence of a similar type orbit in the rectangular four-body problem have recently proved [6], but the existence of such solutions other than those has not been proved. Our result includes and generalizes those results. For example it is shown to exist the Schubart orbit without equal masses, and periodic orbits of the isosceles three-body problem and the collinear symmetric four-body problem.

The $n$-body problem (1) is equivalent to the variational problem with respect to the action functional

$$
\mathcal{A}(\gamma)=\int_{0}^{T} L(\gamma(t), \dot{\gamma}(t)) d t
$$

where the Lagrangian is

$$
L(q, \dot{q})=\frac{1}{2} \sum_{k=1}^{n} m_{k}\left\|\dot{q}_{k}\right\|^{2}+\sum_{i<j} \frac{m_{i} m_{j}}{\left\|q_{i}-q_{j}\right\|} .
$$

Denote the configuration space with the center of masses fixed at the origin by $\mathcal{X}$ :

$$
\mathcal{X}=\left\{q=\left(q_{1}, \ldots, q_{n}\right) \in\left(\mathbb{R}^{d}\right)^{n} \mid \sum_{i=1}^{n} m_{i} q_{i}=0\right\} .
$$

Moreover let $\Lambda=H^{2}(\mathbb{R} / T \mathbb{Z}, \mathcal{X})$ be the Sobolev space of the $L^{2}$ loops $\mathbb{R} / T \mathbb{Z} \rightarrow \mathcal{X}$ with $L^{2}$ derivative.

Let $G$ be a finite group and

$$
\begin{aligned}
& \rho: G \rightarrow O(d), \\
& \sigma: G \rightarrow \mathfrak{S}_{n}
\end{aligned}
$$

be homomorphisms where $O(d)$ is the orthogonal group of degree $d$ and $\mathfrak{S}_{n}$ is the symmetric group of degree $n$. We define the action of $G$ to $\mathcal{X}$ by

$$
g \cdot\left(q_{1}, \ldots, q_{n}\right)=\left(\rho(g) q_{\sigma\left(g^{-1}\right)(1)}, \ldots, \rho(g) q_{\sigma\left(g^{-1}\right)(n)}\right)
$$

for $g \in G$ and $q=\left(q_{1}, \ldots, q_{n}\right) \in \mathcal{X}$. We denote the set of invariant points of $\mathcal{X}$ under $G$ by $\mathcal{X}^{G}$ :

$$
\mathcal{X}^{G}=\{q \in \mathcal{X} \mid g \cdot q=q\} .
$$

Let $\Delta$ be the set of configurations with a collision, i. e. $\Delta:=\bigcup_{i<j} \Delta_{i j}$ where $\Delta_{i j}=\left\{x \in \mathcal{X} \mid x_{i}=x_{j}\right\}$. We assume that $\mathcal{X}^{G} \not \subset \Delta$ through this paper. The curves in $\Lambda^{G}=H^{1}\left(\mathbb{R} / T \mathbb{Z}, \mathcal{X}^{G}\right)$, are the curves fixed by $G$. Let $\mathcal{A}^{G}$ denote the restriction of the action functional $\mathcal{A}$ to $\Lambda^{G}$ :

$$
\mathcal{A}^{G}: \Lambda^{G} \rightarrow \mathbb{R} \cup \infty .
$$

Proposition 1 ([7]). If $\mathcal{A}$ is invariant under the group action of $G$, then a critical point of $\mathcal{A}^{G}$ is a critical point of $\mathcal{A}$. 
Note that if the Lagrangian $L$ is invariant under the group action of $G$ (i.e. $L(g \cdot \dot{q}, g \cdot q)=L(q, \dot{q})$ for all $(q, \dot{q}) \in T \mathcal{X}$ and $g \in G)$, so is $\mathcal{A}$.

In this paper we study the case that $\mathcal{X}^{G}$ is two dimensional. We will first determine a normal form of the Lagrangians $L^{G}=\left.L\right|_{T \mathcal{X}^{G}}$ as follows:

Proposition 2. Assume that $\mathcal{X}^{G}$ is two dimensional. Then there are linear coordinates $(x, y)$ of $\mathcal{X}^{G}$ such that

$$
L^{G}(x, y, \dot{x}, \dot{y})=K(\dot{x}, \dot{y})+U(x, y)
$$

where

$$
\begin{aligned}
K(\dot{x}, \dot{y}) & =\frac{1}{2}\left(\dot{x}^{2}+\dot{y}^{2}\right) \\
U(r \cos \theta, r \sin \theta) & =\frac{1}{r}\left(\sum_{i=1}^{l} \frac{b_{i}}{\left|\sin \left(\theta-c_{i}\right)\right|}+g(\theta)\right) .
\end{aligned}
$$

Here $b_{i}>0,0=c_{1}<c_{2}<\cdots<c_{l}<\pi$ are constants and $g(\theta)$ is $\pi$-periodic smooth function without a singularity. Furthermore if $d=1, g(\theta) \equiv 0$.

As we will describe in Subsection 3.4, the singularities

$$
B_{j}=\left\{\left(r \cos c_{j}, r \sin c_{j}\right) \mid r>0\right\}
$$

are regularizable. For $j=1, \ldots, l$, we denote the region between $B_{j}$ and $B_{j+1}$ by $C_{j}$ :

$$
C_{j}=\left\{(r \cos \theta, r \sin \theta) \mid r>0, c_{j} \leqq \theta \leqq c_{j+1}\right\}
$$

where $c_{l+1}=\pi$. Our main results are the following two theorems.

Theorem 1. Fix any positive constant $T>0$. If

$\nabla U(\cos \theta, \sin \theta) \cdot\left(\cos c_{j}, \sin c_{j}\right)<0, \quad \nabla U(\cos \theta, \sin \theta) \cdot\left(\cos c_{j+1}, \sin c_{j+1}\right)<0$

for $c_{j}<\theta<c_{j+1}$, there exists a periodic solution $\gamma(t)$ of the subsystem such that

1. $\gamma(t)=\gamma(-t), \gamma(t+T)=\gamma(t)$;

2. $\gamma(0) \in B_{j}$ and $\gamma\left(\frac{T}{2}\right) \in B_{j+1}$;

3. $\gamma$ is collision-free except at $t=\frac{T k}{2}(k \in \mathbb{Z})$;

4. $\theta(t)$ is monotone on $[0, T / 2]$ where $\gamma(t)=r(t)(\cos \theta(t), \sin \theta(t))$;

5. $\gamma(t)$ is smooth under the regularizations of $B_{j}$ and $B_{j+1}$.

Corollary 1. Fix any positive constant $T>0$. If $d=1$, there is a periodic solution of the subsystem satisfying properties 1-5 of Theorem 1. 
Proof. As we stated in the last part of Proposition 2, $g(\theta) \equiv 0$. Let $(x, y)=$ $r(\cos \theta, \sin \theta)$. Note that for $c_{j}<\theta<c_{j+1}$,

$$
U(r \cos \theta, r \sin \theta)=\frac{1}{r}\left(\sum_{i=1}^{j} \frac{b_{i}}{\sin \left(\theta-c_{i}\right)}-\sum_{i=j+1}^{l} \frac{b_{i}}{\sin \left(\theta-c_{i}\right)}\right) .
$$

From an easy calculation, it turns out that

$$
\begin{aligned}
& \frac{\partial U}{\partial x}(\cos \theta, \sin \theta)=\sum_{i=1}^{j} \frac{b_{i} \sin c_{i}}{\sin ^{2}\left(\theta-c_{i}\right)}-\sum_{i=j+1}^{l} \frac{b_{i} \sin c_{i}}{\sin ^{2}\left(\theta-c_{i}\right)} \\
& \frac{\partial U}{\partial y}(\cos \theta, \sin \theta)=-\sum_{i=1}^{j} \frac{b_{i} \cos c_{i}}{\sin ^{2}\left(\theta-c_{i}\right)}+\sum_{i=j+1}^{l} \frac{b_{i} \cos c_{i}}{\sin ^{2}\left(\theta-c_{i}\right)}
\end{aligned}
$$

Hence

$$
\begin{aligned}
& \nabla U(\cos \theta, \sin \theta) \cdot\left(\cos c_{j}, \sin c_{j}\right) \\
& =\sum_{i=1}^{j} \frac{b_{i} \sin \left(c_{i}-c_{j}\right)}{r^{2} \sin ^{2}\left(\theta-c_{i}\right)}-\sum_{i=j+1}^{l} \frac{b_{i} \sin \left(c_{i}-c_{j}\right)}{r^{2} \sin ^{2}\left(\theta-c_{i}\right)}<0 \\
& \nabla U(\cos \theta, \sin \theta) \cdot\left(\cos c_{j+1}, \sin c_{j+1}\right) \\
& =\sum_{i=1}^{j} \frac{b_{i} \sin \left(c_{i}-c_{j+1}\right)}{r^{2} \sin ^{2}\left(\theta-c_{i}\right)}-\sum_{i=j+1}^{l} \frac{b_{i} \sin \left(c_{i}-c_{j+1}\right)}{r^{2} \sin ^{2}\left(\theta-c_{i}\right)}<0 .
\end{aligned}
$$

Consequently the assumption (4) is satisfied and this corollary is reduced to Theorem 1.

Theorem 2. Fix any positive constant $T>0$. If

$$
U\left(\cos \left(c_{j}+c_{j+1}-\theta\right), \sin \left(c_{j}+c_{j+1}-\theta\right)\right)=U(\cos \theta, \sin \theta)
$$

and

$$
\nabla U(\cos \theta, \sin \theta) \cdot\left(\cos c_{j}, \sin c_{j}\right)<0
$$

for $c_{j}<\theta<\frac{c_{j}+c_{j+1}}{2}$, then there is a periodic solution $\gamma$ with the properties $1-5$ of Theorem 1 and

$$
\rho\left(\frac{T}{4}+t\right)+\rho\left(\frac{T}{4}-t\right)=c_{j}+c_{j+1}
$$

where $\gamma(t)=r(t)(\cos \rho(t), \sin \rho(t))$.

Corollary 2. Fix any positive constant $T>0$. If $d=1$ and (5) are satisfied, there is a periodic solution of the subsystem satisfying properties 1-5 of Theorem 1 and (7). 
The proof of this corollary is similarly reduced to Theorem 2.

Remark 1. When both Theorem 1 and 2 can be applied, we do not know whether the solutions obtained from these theorems are same.

The paper is organized as follows. In next section we prove Proposition 2. Section 3 is devoted to the proof of theorems. A number of examples are given in the last section.

\section{Proof of Proposition 2}

The kinetic part $K$ on $T \mathcal{X}^{G}$ is a positive definite 2-form of the velocity vector with constant coefficients. Therefore by normalizing this through a linear transformation, the kinetic part can be expressed as

$$
K=\frac{1}{2}\left(\dot{x}^{2}+\dot{y}^{2}\right)
$$

We will next investigate the potential part. Let $q_{i}=\left(x_{i k}\right)_{k=1}^{d}$ and $(x, y)=$ $r(\cos \theta, \sin \theta)$. Since $(x, y)$ are the linear coordinates, we can denote

$$
x_{i k}=a_{i k} x+b_{i k} y=r\left(a_{i k} \cos \theta+b_{i k} \sin \theta\right),
$$

where $a_{i k}$ and $b_{i k}$ are constants. Thus we get

$$
\begin{aligned}
\left|q_{i}-q_{j}\right| & =\sqrt{\sum_{k=1}^{d}\left(x_{i k}-x_{j k}\right)^{2}} \\
& =\sqrt{\sum_{k=1}^{d}\left(\left(a_{i k}-a_{j k}\right) x+\left(b_{i k}-b_{j k}\right) y\right)^{2}} \\
& =r \sqrt{\sum_{k=1}^{d}\left(\left(a_{i k}-a_{j k}\right) \cos \theta+\left(b_{i k}-b_{j k}\right) \sin \theta\right)^{2}} \\
& =r \sqrt{\sum_{k=1}^{d} \beta_{i j k}^{2} \sin ^{2}\left(\theta-\alpha_{i j k}\right)} .
\end{aligned}
$$

where $\beta_{i j k}$ and $\alpha_{i j k} \in[0, \pi)$ are constants such that

$$
\left(a_{i k}-a_{j k}\right) \cos \theta+\left(b_{i k}-b_{j k}\right) \sin \theta= \pm \beta_{i j k} \sin \left(\theta-\alpha_{i j k}\right) .
$$

Here we fix $i \neq j$. Since $\mathcal{X}^{G} \not \subset \Delta, \beta_{i j k} \neq 0$ for some $1 \leqq k \leqq d$. If $\alpha_{i j k}$ with $\beta_{i j k} \neq 0$ are same for $1 \leqq k \leqq d$, then $\left|q_{i}-q_{j}\right|=r \sqrt{\sum_{k=1}^{d} \beta_{i j k}^{2}}\left|\sin \left(\theta-\alpha_{i j 1}\right)\right|$. In the other cases, $\left|q_{i}-q_{j}\right|$ has no zero and is $\pi$-periodic. 
Hence we can denote the potential part by

$$
U=\frac{1}{r}\left(\sum_{i=1}^{l} \frac{b_{i}}{\left|\sin \left(\theta-c_{i}\right)\right|}+g(\theta)\right)
$$

where $b_{i}$ and $0 \leqq c_{1}<c_{2}<\cdots<c_{l}<\pi$ are constants and $g$ is a smooth function with $\pi$ period. Since $K$ is invariant under the rotation, we can assume $c_{1}=0$. This completes the proof of proposition 2 .

From this proof, it turns out that if $d=1$, then $g(\theta) \equiv 0$.

\section{Proof of Theorems}

\subsection{Variational Method}

The solution desired in Theorem 1 will be obtained as a minimizer of $\mathcal{A}^{G}$.

We restrict the domain of $\mathcal{A}^{G}$ to

$$
\Omega_{j}=\left\{\gamma \in H^{1}\left([0, T], C_{j}\right) \mid \gamma(0) \in B_{j}, \gamma(T / 2) \in B_{j+1}\right\} .
$$

The existence of a minimizer of the action functional $\left.\mathcal{A}^{G}\right|_{\Omega_{j}}$ on the weak closure of $\Omega_{j}$ follows from the standard argument (see for example [2, Section 4]).

\subsection{The Exclusion of the Total Collision}

We now prove the minimizer has no total collision. The method below is not new, and almost same as one used in [11, Section 4] and others. It is known that the collisions of minimizer are isolated(see for example [4, Section 5]). Assume that the minimizer $\gamma$ has a total collision at $t_{0}$. By constructing a modified curve with lower value of the action functional, we will show that $\gamma$ must not be a minimizer. We can assume $j=1$ without loss of generality. The Sundman estimates give

$$
\gamma(t)=\left(t-t_{0}\right)^{2 / 3}\left(c_{x}, c_{y}\right)+O\left(\left(t-t_{0}\right)\right)
$$

as $t \rightarrow t_{0}+0$ or $t \rightarrow t_{0}-0$.

We will first consider the case of $t_{0}=0$. Define a function $\delta_{\varepsilon}$ for small $\varepsilon>0$ by

$$
\delta_{\varepsilon}= \begin{cases}\left(\varepsilon^{2}, 0\right) & \text { if } t \in\left[0, \varepsilon^{4}\right] \\ \left(-\varepsilon\left(t-\varepsilon^{4}\right)+\varepsilon^{2}, 0\right) & \text { if } t \in\left[\varepsilon^{4}, \varepsilon^{4}+\varepsilon\right] \\ (0,0) & \text { if } t \in\left[\varepsilon^{4}+\varepsilon, T\right]\end{cases}
$$

and consider the modified curve $\gamma+\delta_{\varepsilon}$. We will compare the values of the action functional for the modified curve and the original one. We split the difference as follows:

$$
\mathcal{A}^{G}\left(\gamma+\delta_{\varepsilon}\right)-\mathcal{A}^{G}(\gamma)=A_{1}+A_{2}+A_{3}
$$


where

$$
\begin{aligned}
& A_{1}=\int_{0}^{\varepsilon^{4}} U\left(\gamma+\delta_{\varepsilon}\right)-U(\gamma) d t \\
& A_{2}=\int_{\varepsilon^{4}}^{\varepsilon^{4}+\varepsilon} U\left(\gamma+\delta_{\varepsilon}\right)-U(\gamma) d t \\
& A_{3}=\frac{1}{2} \int_{\varepsilon^{4}}^{\varepsilon^{4}+\varepsilon}\left(\dot{\gamma}+\dot{\delta}_{\varepsilon}\right)^{2}-\dot{\gamma}^{2} d t .
\end{aligned}
$$

We will investigate each part. Note that the $y$-component are same between $\gamma$ and $\gamma+\delta_{\varepsilon}$. We define a function $W$ by

$$
W(x, y)=U(x, y)-\frac{b_{1}}{|y|} .
$$

The function $W$ is not singular on $B_{1}$. Since $t \in\left[0, \varepsilon^{4}\right]$

$$
\begin{aligned}
\gamma & (t)=t^{2 / 3}\left(c_{x}, c_{y}\right)+O(t), \quad\left(\gamma+\delta_{\varepsilon}\right)(t)=\varepsilon^{2}(1,0)+O\left(t^{2 / 3}\right), \\
A_{1}= & \int_{0}^{\varepsilon^{4}} W\left(\varepsilon^{2}(1,0)+O\left(t^{2 / 3}\right)\right)-W\left(t^{2 / 3}\left(c_{x}, c_{y}\right)+O(t)\right) d t \\
& =\int_{0}^{\varepsilon^{4}} \varepsilon^{-2} W\left((1,0)+O\left(\varepsilon^{-2} t^{2 / 3}\right)\right)-t^{-2 / 3} W\left(\left(c_{x}, c_{y}\right)+O\left(t^{1 / 3}\right)\right) d t \\
& =\int_{0}^{\varepsilon^{4}} \varepsilon^{-2}\left(W(1,0)+O\left(\varepsilon^{-2} t^{2 / 3}\right)\right)-t^{-2 / 3}\left(W\left(c_{x}, c_{y}\right)+O\left(t^{1 / 3}\right)\right) d t \\
& =\int_{0}^{\varepsilon^{4}} \varepsilon^{-2} W(1,0)-t^{-2 / 3} W\left(c_{x}, c_{y}\right)+O\left(\varepsilon^{-4} t^{2 / 3}\right)+O\left(t^{-1 / 3}\right) d t \\
& =\left[\varepsilon^{-2} t W(1,0)-3 t^{1 / 3} W\left(c_{x}, c_{y}\right)+O\left(\varepsilon^{-4} t^{5 / 3}\right)+O\left(t^{2 / 3}\right)\right]_{0}^{\varepsilon^{4}} \\
& =-3 W(c) \varepsilon^{4 / 3}+O\left(\varepsilon^{2}\right) .
\end{aligned}
$$

We next estimate $A_{2}$ by using the assumption (4), which is

$$
\frac{\partial U}{\partial x}<0
$$

in this case. From this assumption, it follows that

$$
U\left(\left(\gamma+\delta_{\varepsilon}\right)(t)\right)-U(\gamma(t))<0
$$

for $t \in\left[\varepsilon^{4}, \varepsilon^{4}+\varepsilon\right]$. Hence $A_{2}<0$. 
Finally we compute $A_{3}$ as follows:

$$
\begin{aligned}
A_{3} & =\frac{1}{2} \int_{\varepsilon^{4}}^{\varepsilon^{4}+\varepsilon} 2 \dot{\gamma} \dot{\delta}_{\varepsilon}+\dot{\delta}_{\varepsilon}^{2} d t \\
& =\frac{1}{2} \int_{\varepsilon^{4}}^{\varepsilon^{4}+\varepsilon} 2 \cdot \frac{2}{3} t^{-1 / 3} c_{x}(-\varepsilon)+O\left(t^{2 / 3}\right)+\varepsilon^{2} d t \\
& =-c_{x} \varepsilon\left(\left(\varepsilon^{4}+\varepsilon\right)^{2 / 3}-\varepsilon^{8 / 3}\right)+O\left(\varepsilon^{5 / 3}\right)+\frac{1}{2} \varepsilon^{3} \\
& =-c_{x} \varepsilon^{5 / 3}\left(\left(\varepsilon^{3}+1\right)^{2 / 3}-\varepsilon^{2}\right)+O\left(\varepsilon^{5 / 3}\right) \\
& =-c_{x} \varepsilon^{\frac{5}{3}}+O\left(\varepsilon^{5 / 3}\right) .
\end{aligned}
$$

Consequently we get

$$
A_{1}+A_{2}+A_{3}=-3 W(c) \varepsilon^{4 / 3}+O\left(\varepsilon^{5 / 3}\right),
$$

and hence (9) is negative for small $\varepsilon>0$.

In the case that $\gamma(t)$ has a total collision at $t_{0}=T$, we similarly make a modified curve with a lower value of the action functional than one of $\gamma(t)$.

The case of $t_{0} \in(0, T)$ is a little more complicated. We can make a modified curve with a lower value of the action functional as follows:

$$
\gamma_{\varepsilon}(t)= \begin{cases}\gamma(t)+\delta_{\varepsilon}\left(t_{0}-t\right) & 0 \leqq t \leqq t_{0} \\ \gamma(t)+\delta_{\varepsilon}\left(t-t_{0}\right) & t_{0} \leqq t \leqq T\end{cases}
$$

for small $\varepsilon$. This completes the proof that the minimizer has no total collision. Consequently the minimizer belongs to $\Omega_{j}$ and hence the minimizer satisfies the property 2 of Theorem 1 .

\subsection{The Monotonicity of the Argument and the Exclusion of the Extra Partial Collisions}

Let $\gamma$ be a minimizer and $\gamma(t)=r(t)(\cos \theta(t), \sin \theta(t))$. From the result $(\gamma(t) \neq$ 0 for any $t$ ) of the proceeding subsection, $\theta(t)$ is well-defined and $\theta(0)=$ $c_{j}, \theta(T / 2)=c_{j+1}$. In this subsection we will prove the monotonicity of $\theta$ and the absence of extra partial collisions. This proof is different from one of [11].

Proposition 3. $\theta(t)$ is monotonically increasing on $t \in[0, T / 2]$.

Proof. From the setting (see (3) and (8)) of the domain of $\mathcal{A}^{G}$, for any $t \in$ $[0, T / 2], \gamma(t)$ belongs to $C_{j}$, that is, $c_{j} \leqq \theta(t) \leqq c_{j+1}$.

We will first show that $\ddot{\theta}(t)$ is non-zero when $\dot{\theta}(t)=0$. Let $V(\theta)=U(\cos \theta, \sin \theta)$. The Lagrangian in the polar coordinates is

$$
L(r, \theta, \dot{r}, \dot{\theta})=\frac{1}{2}\left(\dot{r}^{2}+r^{2} \dot{\theta}^{2}\right)+r^{-1} V(\theta) .
$$


Through the Legendre transformation, the corresponding Hamiltonian is

$$
H\left(p_{r}, p_{\theta}, r, \theta\right)=\frac{1}{2}\left(p_{r}^{2}+r^{-2} p_{\theta}^{2}\right)-r^{-1} V(\theta)
$$

where $p_{r}=\dot{r}, p_{\theta}=r^{2} \dot{\theta}$. The canonical equations are

$$
\begin{aligned}
\dot{r} & =p_{r} \\
\dot{\theta} & =r^{-2} p_{\theta} \\
\dot{p}_{r} & =r^{-3} p_{\theta}^{2}-r^{-2} V(\theta) \\
\dot{p}_{\theta} & =r^{-1} V^{\prime}(\theta) .
\end{aligned}
$$

Assume that $c_{j}<\theta\left(t_{0}\right)<c_{j+1}$ and $\dot{\theta}\left(t_{0}\right)=\ddot{\theta}\left(t_{0}\right)=0$ for some $t_{0} \in[0, T / 2]$. Since $\dot{p}_{\theta}\left(t_{0}\right)=0, V^{\prime}\left(\theta_{0}\right)=0$ where $\theta_{0}=\theta\left(t_{0}\right)$. The set $\left\{\left(p_{r}, p_{\theta}, r, \theta\right) \mid p_{\theta}=\right.$ $\left.0, \theta=\theta_{0}\right\}$ is an invariant set of (11). This solution passes the set. Because of the unicity of solutions of ordinary differential equations, this solution holds $\theta(t)=\theta_{0}$ for $t \in[0, T / 2]$. This contradicts the fact that $\theta(0)=c_{j}, \theta(T)=c_{j+1}$. Hence each critical point of $\theta(t)$ is a local minimum or maximum.

On the other hand, if $\theta\left(t_{0}\right)=c_{j}$ ( or $c_{j+1}$ ) and $\theta(t)$ are locally constant near $t=t_{0}$, then the value of the action functional is infinite. Hence $\theta(t)$ is not constant in any partial interval.

We will next prove the monotonicity of $\theta(t)$. Assume that $\theta(t)$ is not monotonically increasing. Here this also includes the case where the extra partial collision occur(see Figure 2).

Let $t_{1}$ be the smallest local maximum point of $\theta(t)$ and $\theta_{\max }$ be its maximum value:

$$
\begin{aligned}
t_{1} & =\min \{t>0 \mid t \text { is a local maximum point of } \theta(t)\} \\
\theta_{\max } & =\theta\left(t_{1}\right)
\end{aligned}
$$

From the intermediate value theorem, we can define $t_{2}$ by

$$
t_{2}=\min \left\{t>t_{1} \mid \theta(t)=\theta_{\max }\right\}
$$

in the case of $\theta_{\max }<c_{j+1}$, while we let $t_{2}=T / 2$ in the case of $\theta_{\max }=c_{j+1}$. Now we can uniquely determine $\theta_{\min }, V_{\min }, \theta_{0}, s_{1}$ and $s_{2}$ as follows:

$$
\begin{aligned}
\theta_{\min } & =\min \left\{\theta(t) \mid t_{1}<t<t_{2}\right\} \\
V_{\min } & =\min \left\{V(\theta) \mid \theta_{\min } \leqq \theta \leqq \theta_{\max }\right\} \\
\theta_{0} & =\max \left\{\theta \in\left[\theta_{\min }, \theta_{\max }\right] \mid V(\theta)=V_{\min }\right\} \\
s_{1} & =\max \left\{t<t_{1} \mid \theta(t)=\theta_{0}\right\} \\
s_{2} & =\max \left\{t<t_{2} \mid \theta(t)=\theta_{0}\right\} .
\end{aligned}
$$

We construct a modified curve $\gamma_{*}(t)=r(t)\left(\cos \theta_{*}(t), \sin \theta_{*}(t)\right)$ by letting 


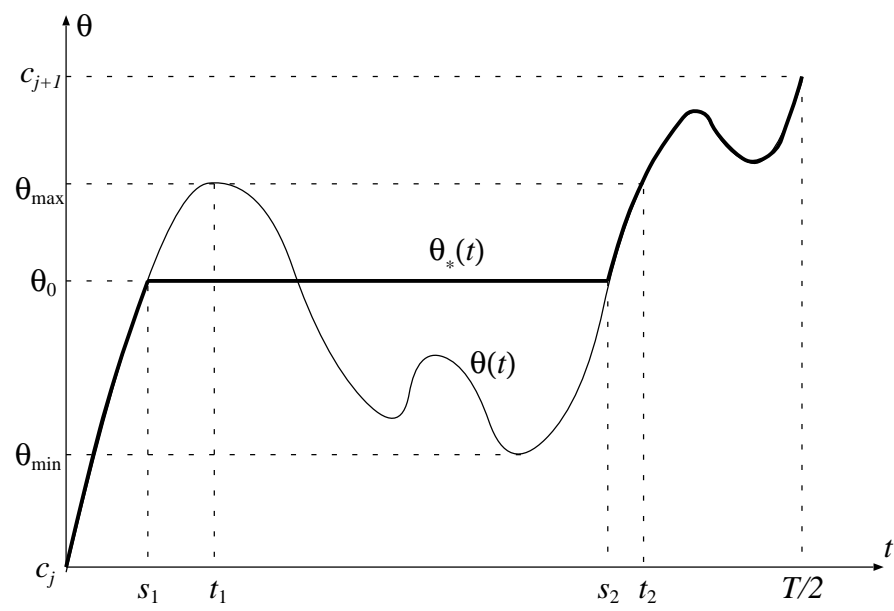

Figure 1: Non-monotonic curve

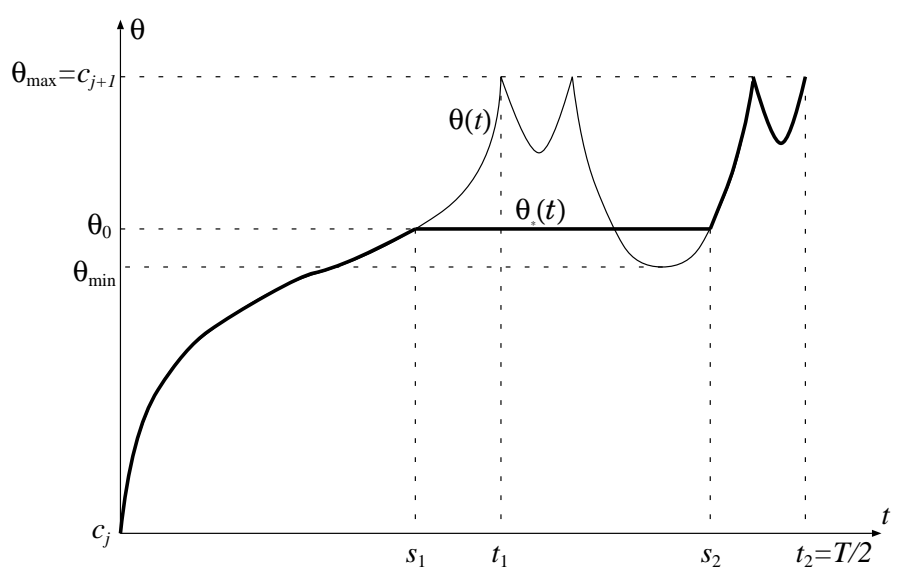

Figure 2: Curve with a extra partial collision 


$$
\theta_{*}(t)= \begin{cases}\theta(t) & \text { for } t \leqq s_{1} \text { or } t \geqq s_{2} \\ \theta_{0} & \text { for } s_{1}<t<s_{2}\end{cases}
$$

Then the difference of their values of the action functional is

$$
\mathcal{A}^{G}\left(\gamma_{*}\right)-\mathcal{A}^{G}(\gamma)=\int_{s_{1}}^{s_{2}}-\frac{1}{2} r^{2}(t) \dot{\theta}^{2}(t)+r^{-1}(t)\left(V_{\min }-V(\theta(t))\right) d t<0 .
$$

This is a contradiction and completes the proof. This argument implies that the minimizer has no extra partial collision.

This proposition shows the property 3 and 4 in Theorem 1 .

\subsection{Regularizability of the Partial Collisions}

We assert that the derivative $\dot{\gamma}(t)$ of the minimizer is perpendicular to the boundary lines $B_{j}$ and $B_{j+1}$ (see Figure 3 ):

\section{Proposition 4.}

$$
\left(\lim _{t \rightarrow+0} \frac{\dot{\gamma}(t)}{\|\dot{\gamma}(t)\|}\right) \perp B_{j}, \quad\left(\lim _{t \rightarrow T / 2-0} \frac{\dot{\gamma}(t)}{\|\dot{\gamma}(t)\|}\right) \perp B_{j+1}
$$

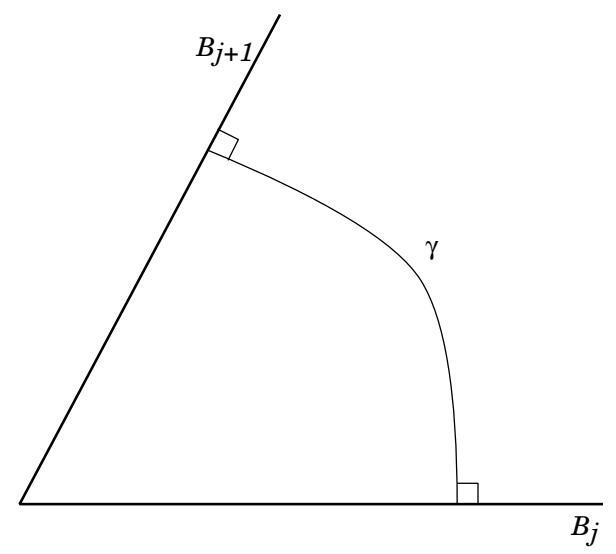

Figure 3: Symmetric minimizer

Proof. The minimizer $\gamma$ satisfies the first variational formula:

$$
\delta \mathcal{A}(\gamma)=\left[\frac{\partial L}{\partial \dot{\mathbf{q}}} \cdot \delta \gamma\right]_{t=0}^{T / 2}-\int_{0}^{T / 2}\left(\frac{d}{d t} \frac{\partial L}{\partial \dot{\mathbf{q}}}-\frac{\partial L}{\partial \mathbf{q}}\right) \cdot \delta \gamma d t=0
$$


for any $\delta \gamma \in T \Omega_{j}$. Hence by considering any variations $\delta \gamma$ with $\delta \gamma(t)=0$ near $t=0$ and $T / 2$, we see that the minimizer satisfies the Euler-Lagrange equation:

$$
\frac{d}{d t} \frac{\partial L}{\partial \dot{\mathbf{q}}}-\frac{\partial L}{\partial \mathbf{q}}=0
$$

We again consider (13) for $\delta \gamma$ with $\delta \gamma(t)=0$ near $t=T / 2$, and then we obtain

$$
\lim _{t \rightarrow+0} \frac{\partial L}{\partial \dot{\mathbf{q}}}(\gamma(t), \dot{\gamma}(t)) \cdot \delta \gamma(t)=0
$$

that is

$$
\lim _{t \rightarrow+0} \dot{\gamma}(t) \cdot \delta \gamma(t)=0
$$

Note that $\|\dot{\gamma}(t)\|^{-1} \rightarrow 0$ as $t \rightarrow+0$ from the conservation law of the energy. Thus we have

$$
\lim _{t \rightarrow+0} \frac{\dot{\gamma}(t)}{\|\dot{\gamma}(t)\|} \cdot \delta \gamma(t)=0
$$

Since $\delta \gamma(0)$ can have any point in $T B_{j}$ it follows that

$$
\left(\lim _{t \rightarrow+0} \frac{\dot{\gamma}(t)}{\|\dot{\gamma}(t)\|}\right) \perp B_{j}
$$

We can similarly show that

$$
\left(\lim _{t \rightarrow T / 2-0} \frac{\dot{\gamma}(t)}{\|\dot{\gamma}(t)\|}\right) \perp B_{j+1}
$$

We will regularize the binary collisions through the Levi-Civita transformation. We assume $j=1$ without loss of generality. Our equations are

$$
\dot{x}=p_{x}, \quad \dot{y}=p_{y}, \quad \dot{p_{x}}=\frac{\partial W}{\partial x}(x, y), \quad \dot{p_{y}}=-\frac{b_{1} y}{|y|^{3}}+\frac{\partial W}{\partial y}(x, y)
$$

where $W$ is defined by (10). We can regularize the partial collisions occurring at $B_{1}$ as follows. Letting $y=w^{2} / 2, p_{y}=p_{w} / w$, the equations are

$\dot{x}=p_{x}, \quad \dot{w}=\frac{p_{w}}{w^{2}}, \quad \dot{p_{x}}=\frac{\partial W}{\partial x}\left(x, \frac{w^{2}}{2}\right), \quad \dot{p_{w}}=\frac{p_{w}^{2}}{w^{3}}-\frac{4 b_{1}}{w^{3}}+w \frac{\partial W}{\partial y}\left(x, \frac{w^{2}}{2}\right)$.

By using the energy equation

$$
\frac{p_{x}^{2}}{2}+\frac{p_{w}^{2}}{2 w^{2}}-\frac{2 b_{1}}{w^{2}}-W\left(x, \frac{w^{2}}{2}\right)=h
$$

we have

$$
\begin{aligned}
& \dot{x}=p_{x}, \quad \dot{w}=\frac{p_{w}}{w^{2}}, \quad \dot{p_{x}}=\frac{\partial W}{\partial x}\left(x, \frac{w^{2}}{2}\right), \\
& \dot{p_{w}}=-\frac{p_{x}^{2}}{w}+\frac{2}{w} W\left(x, \frac{w^{2}}{2}\right)+\frac{2 h}{w}+w \frac{\partial W}{\partial y}\left(x, \frac{w^{2}}{2}\right) \text {. }
\end{aligned}
$$


Changing the time variable by $d t=w^{2} d \tau$ from the original time $t$ to the new time $\tau$, the equations become

$$
\begin{array}{r}
x^{\prime}=w^{2} p_{x}, \quad w^{\prime}=p_{w}, \quad p_{x}^{\prime}=w^{2} \frac{\partial W}{\partial x}\left(x, \frac{w^{2}}{2}\right), \\
p_{w}^{\prime}=-p_{x}^{2} w+2 w W\left(x, \frac{w^{2}}{2}\right)+2 h w+w^{3} \frac{\partial W}{\partial y}\left(x, \frac{w^{2}}{2}\right)
\end{array}
$$

where' denotes the differentation with respect to $\tau$.

Let

$$
R:\left(p_{x}, p_{w}, x, w\right) \mapsto\left(-p_{x}, p_{w}, x,-w\right) .
$$

The system (15) is reversible with respect to $R$, that is, if $\eta(t)$ is a solution, then so is $R \eta(-t)$. From (12), the minimizer $\gamma$ satisfies $p_{x}(0)=w(0)=0$ in this coordinates, and hence $\gamma(t)$ and $R \gamma(-t)$ are equal at $t=0$. From the unicity of solutions of ordinary differential equations, $\gamma(t)$ can be smoothly connected with $R \gamma(-t)$ at $t=0$ and satisfies $\gamma(-t)=\gamma(t)$. Similarly $\gamma(t)$ can be smoothly connected at $t=T / 2$ under the regularization such that $\gamma(-t+T / 2)=$ $\gamma(t+T / 2)$ and is extended for $t \in \mathbb{R}$. This shows the property 5 in Theorem 1 .

Moreover we have

$$
\gamma(t+T)=\gamma\left(\frac{T}{2}+\left(\frac{T}{2}+t\right)\right)=\gamma\left(\frac{T}{2}-\left(\frac{T}{2}+t\right)\right)=\gamma(-t)=\gamma(t)
$$

Consequently $\gamma$ is a periodic solution with period $T$. This shows the property 1 of Theorem 1 and completes the proof of Theorem 1 .

\subsection{Proof of Theorem 2}

Let

$$
\begin{aligned}
D_{j} & =\left\{r\left(\cos \frac{c_{j}+c_{j+1}}{2}, \sin \frac{c_{j}+c_{j+1}}{2}\right) \mid r>0\right\} \\
\Gamma_{j} & =\left\{\gamma \in H^{1} \mid \gamma(0) \in B_{j}, \gamma(T / 4) \in D_{j}\right\} .
\end{aligned}
$$

The solution desired in Theorem 2 can be obtained as a minimizer of $\left.\mathcal{A}^{G}\right|_{\Gamma_{j}}$. The proof is completely analogous to the proof of Theorem 1 .

\section{Examples}

We will give several examples to which we can apply Propositions 1 and our results. 


\subsection{Collinear Three-Body Problem}

We consider the case of $n=3, d=1$ and $G=\{1\}$, in which the system has two degrees of freedom. Through the Jacobi coordinates:

$$
\begin{aligned}
X & =q_{3}-\frac{1}{m_{1}+m_{2}}\left(m_{1} q_{1}+m_{2} q_{2}\right), \\
Y & =q_{2}-q_{1},
\end{aligned}
$$

the Lagrangian is

$$
\begin{aligned}
L=\frac{1}{2}\left(\frac{m_{3}\left(m_{1}+m_{2}\right)}{m_{1}+m_{2}+m_{3}} \dot{X}^{2}+\frac{m_{1} m_{2}}{m_{1}+m_{2}} \dot{Y}^{2}\right)+\frac{m_{1} m_{2}}{|Y|} \\
+\frac{m_{2} m_{3}\left(m_{1}+m_{2}\right)}{\left|\left(m_{1}+m_{2}\right) X+m_{1} Y\right|}+\frac{m_{1} m_{3}\left(m_{1}+m_{2}\right)}{\left|\left(m_{1}+m_{2}\right) X+m_{2} Y\right|} .
\end{aligned}
$$

By letting

$$
x=\sqrt{\frac{m_{3}\left(m_{1}+m_{2}\right)}{m_{1}+m_{2}+m_{3}}} X, \quad y=\sqrt{\frac{m_{1} m_{2}}{m_{1}+m_{2}}} Y,
$$

the Lagrangian is normalized:

$$
\begin{aligned}
L=\frac{1}{2} \dot{x}^{2}+\frac{1}{2} \dot{y}^{2}+\sqrt{\frac{m_{1}^{3} m_{2}^{3}}{m_{1}+m_{2}}} \frac{1}{|y|} & +\sqrt{\frac{m_{2}^{3} m_{3}^{3}}{m_{2}+m_{3}}} \frac{1}{\left|\left(\sin c_{2}\right) x-\left(\cos c_{2}\right) y\right|} \\
& +\sqrt{\frac{m_{1}^{3} m_{3}^{3}}{m_{1}+m_{3}}} \frac{1}{\left|\left(\sin c_{3}\right) x-\left(\cos c_{3}\right) y\right|},
\end{aligned}
$$

where

$$
\begin{gathered}
c_{2}=\arctan \sqrt{\frac{m_{2}\left(m_{1}+m_{2}+m_{3}\right)}{m_{1} m_{3}},} \\
c_{3}=-\arctan \sqrt{\frac{m_{1}\left(m_{1}+m_{2}+m_{3}\right)}{m_{2} m_{3}} .} .
\end{gathered}
$$

Since $d=1$, we obtain periodic orbit with two binary collisions from Corollary 1. Moreover we obtain a more symmetric periodic orbit in the case of $m_{1}=m_{3}$ from Corollary 2, which is known as Schubart's orbit. As we stated in Section 1, Moeckel [5] and Venturelli [11] have showed the existence of the later orbit.

\subsection{Isosceles Three-Body Problem}

We consider the planar three-body problem with $m_{1}=m_{2}$ and a group $G=<$ $g \mid g^{2}=1>$ such that

$$
\rho(g)=\left(\begin{array}{cc}
1 & 0 \\
0 & -1
\end{array}\right), \quad \sigma(g)=(1,2) .
$$




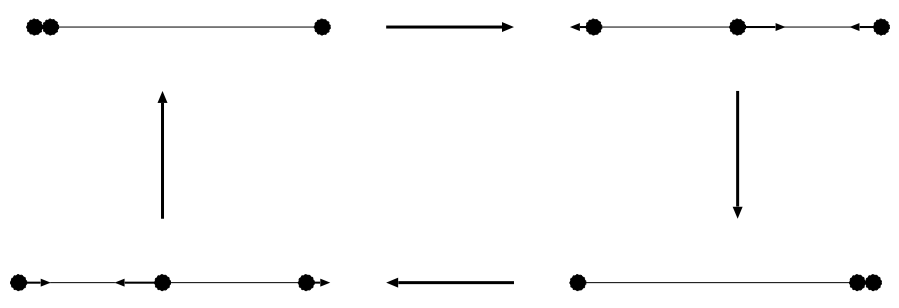

Figure 4: The Schubart's orbit

This subsystem is known as isosceles three-body problem.

Letting

$$
\begin{aligned}
& q_{1}=\left(\sqrt{\left.\frac{m_{3}}{2 m_{1}\left(2 m_{1}+m_{3}\right)} x, \frac{1}{\sqrt{2 m_{1}}} y\right),}\right. \\
& q_{2}=\left(\sqrt{\frac{m_{3}}{2 m_{1}\left(2 m_{1}+m_{3}\right)}} x,-\frac{1}{\sqrt{2 m_{1}}} y\right), \\
& q_{3}=\left(-\sqrt{\frac{2 m_{1}}{m_{3}\left(2 m_{1}+m_{3}\right)}} x, 0\right),
\end{aligned}
$$

we obtain the normalized Lagrangian:

$$
L=\frac{1}{2}\left(\dot{x}^{2}+\dot{y}^{2}\right)+\frac{m^{5 / 2}}{\sqrt{2}|y|}+\frac{\left(2 m_{1} m_{3}\right)^{3 / 2}}{\sqrt{\left(2 m_{1}+m_{3}\right) x^{2}+m_{3} y^{2}}} .
$$

The potential part is

$$
U=\frac{m^{5 / 2}}{\sqrt{2}|y|}+\frac{\left(2 m_{1} m_{3}\right)^{3 / 2}}{\sqrt{\left(2 m_{1}+m_{3}\right) x^{2}+m_{3} y^{2}}},
$$

and its partial derivative with respect to $x$ is

$$
\frac{\partial U}{\partial x}=-\frac{\left(2 m_{1} m_{3}\right)^{3 / 2}\left(2 m_{1}+m_{3}\right) x}{\left(\left(2 m_{1}+m_{3}\right) x^{2}+m_{3} y^{2}\right)^{3 / 2}} .
$$

For $x>0, \frac{\partial U}{\partial x}<0$. Hence $\nabla U(\cos \theta, \sin \theta) \cdot(1,0)<0$ for $0<\theta<\pi / 2$. Therefore we can apply theorem 2 and then obtain periodic orbit which has numerically been found by Broucke [1] (see Figure 5).

\subsection{Collinear Symmetric Four-Body Problem}

We consider the case of $n=4, d=1, m_{1}=m_{2}, m_{3}=m_{4}$ and $G=\left\langle g \mid g^{2}=1\right\rangle$, and define the group action by

$$
\rho(g)=-1, \quad \sigma(g)=\left(\begin{array}{ll}
1 & 2
\end{array}\right)\left(\begin{array}{ll}
3 & 4
\end{array}\right) .
$$




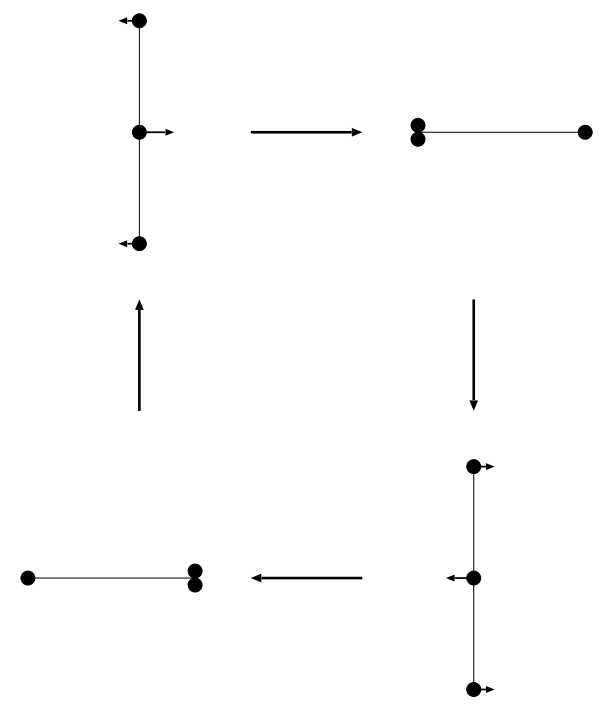

Figure 5: The Broucke's orbit

Letting

$$
q_{1}=-q_{2}=\frac{x}{\sqrt{2 m_{1}}}, \quad q_{3}=-q_{4}=\frac{y}{\sqrt{2 m_{3}}},
$$

we get

$$
L=\frac{1}{2}\left(\dot{x}^{2}+\dot{y}^{2}\right)+\frac{m_{1}^{5 / 2}}{\sqrt{2}|x|}+\frac{m_{3}^{5 / 2}}{\sqrt{2}|y|}+\frac{\sqrt{2} m_{1}^{3 / 2} m_{3}^{3 / 2}}{\left|\sqrt{m_{3}} x-\sqrt{m_{1}} y\right|}+\frac{\sqrt{2} m_{1}^{3 / 2} m_{3}^{3 / 2}}{\left|\sqrt{m_{3}} x+\sqrt{m_{1}} y\right|} .
$$

Since $d=1$, we get symmetric collinear four-body orbit from Corollary 1 , which Sekiguchi [9] have numerically found.

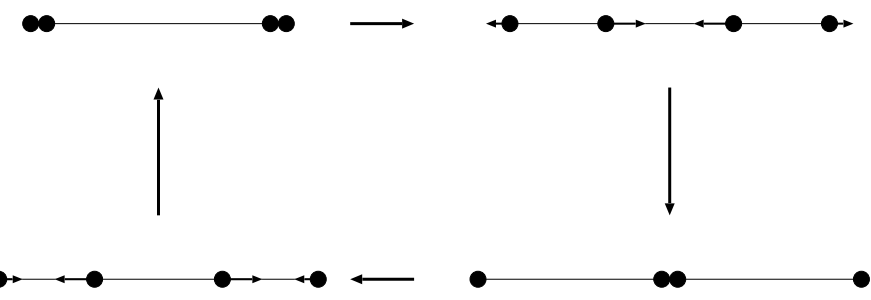

Figure 6: The Sekiguchi's orbit 


\subsection{Rhomboidal Four-Body Problem}

We here consider the four-body problem with rhomboidal symmetry. Let $m_{1}=$ $m_{2}, m_{3}=m_{4}$ and

$$
q_{1}=-q_{2}=\left(\frac{1}{\sqrt{2 m_{1}}} x, 0\right), q_{3}=-q_{4}=\left(0, \frac{1}{\sqrt{2 m_{2}}} y\right) .
$$

The potential part of the normalized Lagrangian is

$$
U=\frac{m_{1}^{5 / 2}}{\sqrt{2}|x|}+\frac{m_{2}^{5 / 2}}{\sqrt{2}|y|}+\frac{4 \sqrt{2} m_{1}^{3 / 2} m_{2}^{3 / 2}}{\sqrt{m_{2} x^{2}+m_{1} y^{2}}} .
$$

Since

$$
\frac{\partial U}{\partial x}=-\frac{m_{1}^{5 / 2}}{\sqrt{2} x^{2}}-\frac{4 \sqrt{2} m_{1}^{3 / 2} m_{2}^{5 / 2} x}{\left(m_{2} x^{2}+m_{1} y^{2}\right)^{3 / 2}}<0
$$

and

$$
\frac{\partial U}{\partial y}=-\frac{m_{2}^{5 / 2}}{\sqrt{2} y^{2}}-\frac{4 \sqrt{2} m_{1}^{5 / 2} m_{2}^{3 / 2} y}{\left(m_{2} x^{2}+m_{1} y^{2}\right)^{3 / 2}}<0
$$

for $x>0$ and $y>0, \nabla U(\cos \theta, \sin \theta) \cdot(1,0)<0$ and $\nabla U(\cos \theta, \sin \theta) \cdot(0,1)<0$ for $0<\theta<\pi / 2$. we can apply theorem 1 and then obtain periodic orbit. If $m_{1}=m_{2}$, we obtain symmetric periodic orbit with the aid of theorem 2 .

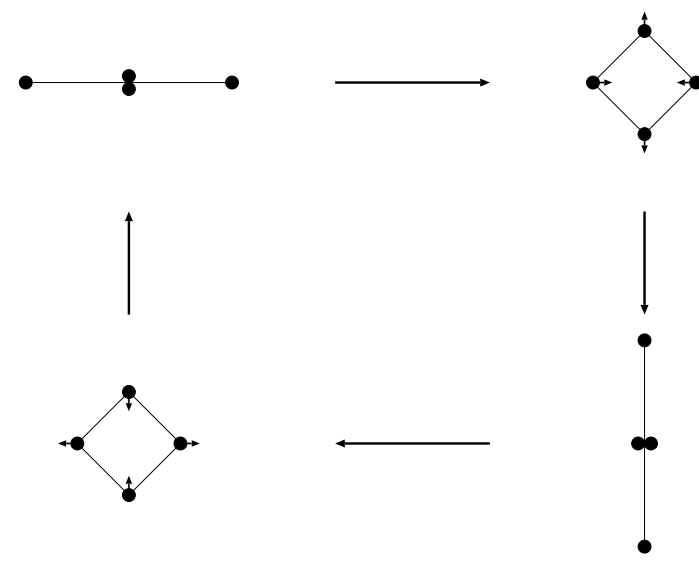

Figure 7: Rhomboidal four-body orbit

\subsection{Rectangular Four-Body Problem}

We consider the four-body problem with the rectangular symmetry: let $m_{1}=$ $m_{2}, m_{3}=m_{4}$ and

$$
q_{1}=(x, y), q_{2}=(x,-y), q_{3}=(-x, y), q_{4}=(-x,-y) .
$$


We can easily check the assumption of Theorem 1 and then obtain periodic orbit. Moreover we can obtain more symmetric orbit from theorem 2 if all masses are equal. The existence of the later orbit have recently already proved by Ouyang, Simmons and Yan [6].

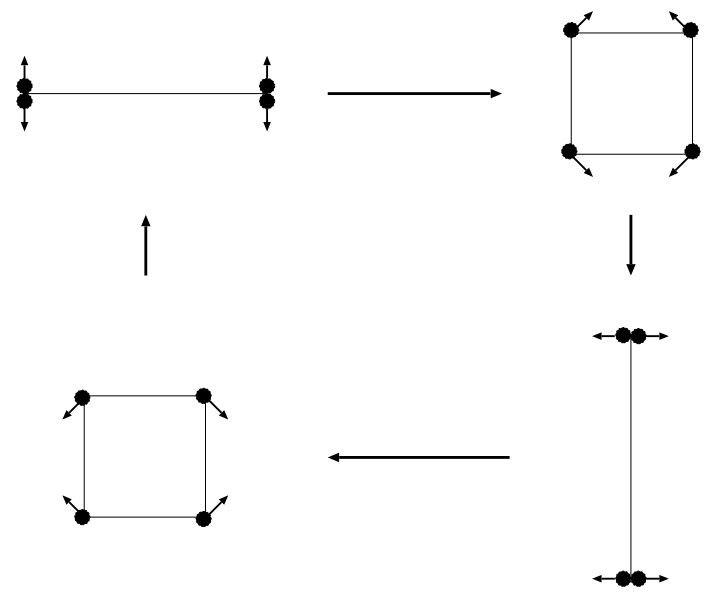

Figure 8: Rectangular four-body orbit

We easily generalize this case to planar $2 n$ - and $2 n+1$-body problem. Let $m_{1}=\cdots=m_{n}, m_{n+1}=\cdots=m_{2 n}, G=D_{n}=<g, c \mid g^{n}=c^{2}=1, g c g=c>$ and

$$
\begin{aligned}
& \rho(g)=\left(\begin{array}{cc}
\cos \frac{2 \pi}{n} & -\sin \frac{2 \pi}{n} \\
\sin \frac{2 \pi}{n} & \cos \frac{2 \pi}{n}
\end{array}\right) \\
& \sigma(g)=\left(\begin{array}{llll}
1 & 2 & \ldots & n
\end{array}\right)(n+1 \quad n+2 \ldots 2 n) \\
& \rho(c)=\left(\begin{array}{cc}
1 & 0 \\
0 & -1
\end{array}\right) \\
& \sigma(c)=(2 n)(3 n-1) \ldots([(n+1) / 2] \quad[(n+4) / 2]) \\
& (n+22 n)(n+32 n-1) \ldots([(3 n+1) / 2][(3 n+4) / 2])
\end{aligned}
$$

where [ ] is the Gaussian symbol. We obtain periodic solution from theorem 1, and symmetric one from theorem 2 if all masses are equal. Moreover we can add one particle fixed at the origin with any mass and hence also get periodic orbits in the $2 n+1$-body problem.

\subsection{Generalized Orbits}

Collisions of more than two particles are not regularizable in general. But as far as we consider the subsystems with two degrees of freedom, the partial collisions are regularizable as we showed in Subsection 3.4. There are also several examples as follows. 
(1) We can extend the Broucke orbit (Subsection 4.2) to $n+1$-body problem with $n$-sided pyramidical symmetry. We consider the $\operatorname{spatial}\left(V=\mathbb{R}^{3}\right) n+1$ body problem with $m_{1}=m_{2}=\cdots=m_{n}$ and cyclic group $G=<g>$ such that

$$
\rho(g)=\left(\begin{array}{ccc}
\cos \frac{2 \pi}{n} & -\sin \frac{2 \pi}{n} & 0 \\
\sin \frac{2 \pi}{n} & \cos \frac{2 \pi}{n} & 0 \\
0 & 0 & 1
\end{array}\right), \quad \sigma(g)=(1,2, \ldots, n) .
$$

We can obtain periodic orbit similarly as Broucke's orbit (Figure 9).
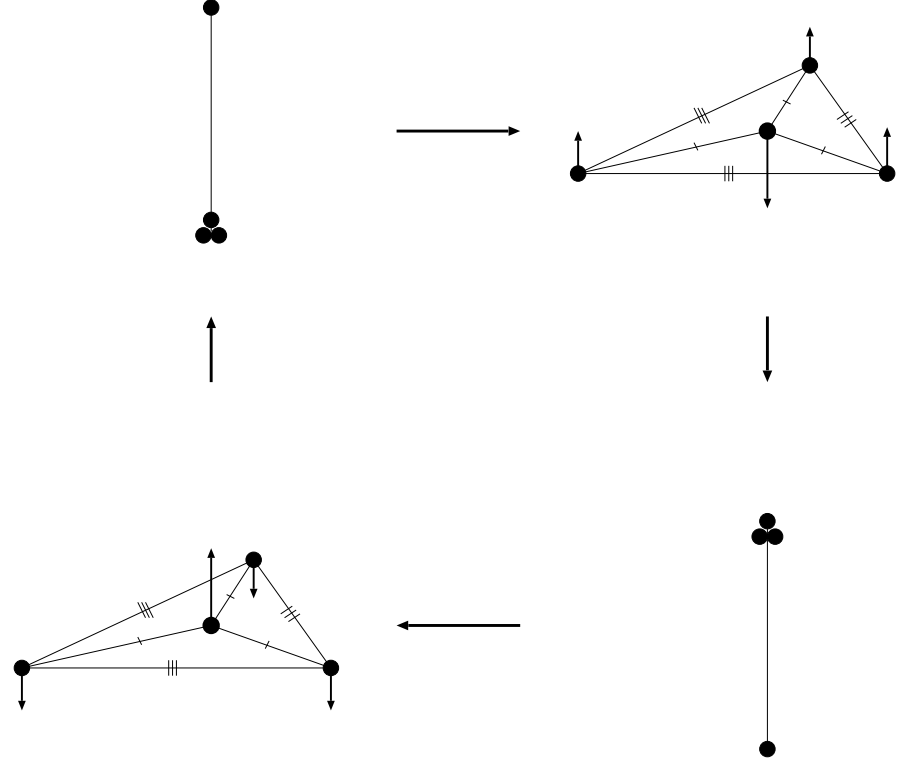

Figure 9: Pyramidal four-body orbit

(2) By adding one more particle fixed at the origin into the Sekiguchi orbit (Subsection 4.3), we can obtain a periodic orbit of symmetric five-body problem (Figure 10).

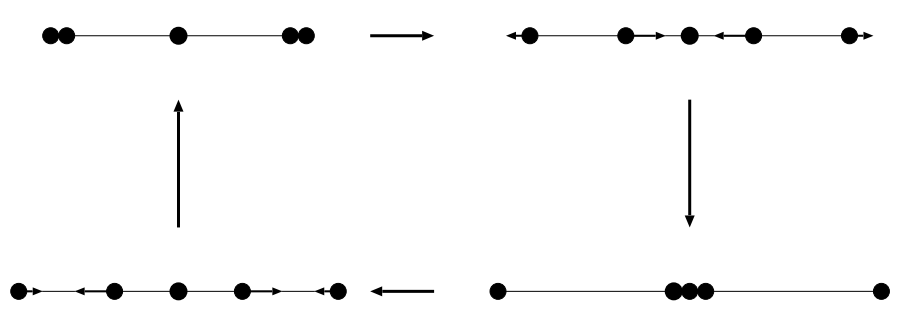

Figure 10: Symmetric collinear five-body orbit 
(3) We also generalize the rectangular orbits to prismatic $2 n$-body problem (Figure 11). Let $d=3, G=D_{n} \times C_{2}, D_{2}=<g, c_{1} \mid g^{n}=c^{2}=1, g c g=c_{1}>$,
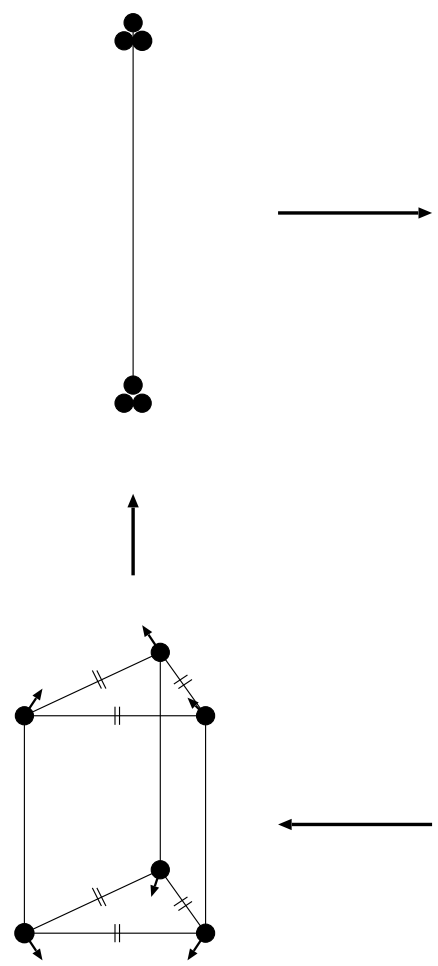

Figure 11: Triangular prismatic six-body orbit 
$C_{2}=<c_{2} \mid c_{2}^{2}=1>$ and

$$
\begin{aligned}
& \rho(g)=\left(\begin{array}{ccc}
1 & 0 & 0 \\
0 & \cos \frac{2 \pi}{n} & -\sin \frac{2 \pi}{n} \\
0 & \sin \frac{2 \pi}{n} & \cos \frac{2 \pi}{n}
\end{array}\right) \\
& \sigma(g)=\left(\begin{array}{llll}
1 & 2 & \ldots & n
\end{array}\right)(n+1 \quad n+2 \ldots .2 n) \\
& \rho\left(c_{1}\right)=\left(\begin{array}{ccc}
1 & 0 & 0 \\
0 & -1 & 0 \\
0 & 0 & 1
\end{array}\right) \\
& \sigma\left(c_{1}\right)=(2 n)(3 n-1) \ldots([(n+1) / 2][(n+4) / 2]) \\
& (n+22 n)(n+32 n-1) \ldots([(3 n+1) / 2][(3 n+4) / 2]) \\
& \rho\left(c_{2}\right)=\left(\begin{array}{ccc}
-1 & 0 & 0 \\
0 & 1 & 0 \\
0 & 0 & 1
\end{array}\right) \\
& \sigma\left(c_{2}\right)=(1 \quad n+1)(2 n+2) \ldots\left(\begin{array}{ll}
n & 2 n
\end{array}\right) \text {. }
\end{aligned}
$$

Furthermore we can add one particle fixed at the origin and then obtain periodic orbit in the $2 n+1$-body problem.

(4) As exotic examples we consider orbits whose configuration consists of two similar polyhedrons whose behavior is like the Sekiguchi orbit (see Figure 12).

(5) Furthermore we can consider orbits whose configuration consists of two dual
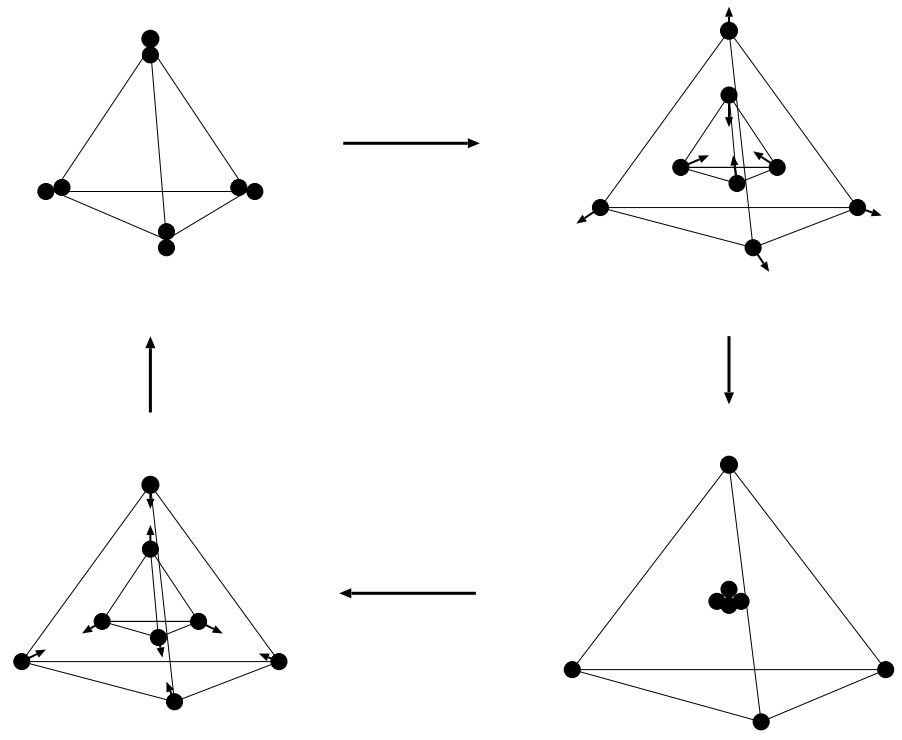

Figure 12: Eight-body orbit whose configuration consists of two similar regular tetrahedrons

polyhedrons (see Figure 13). 

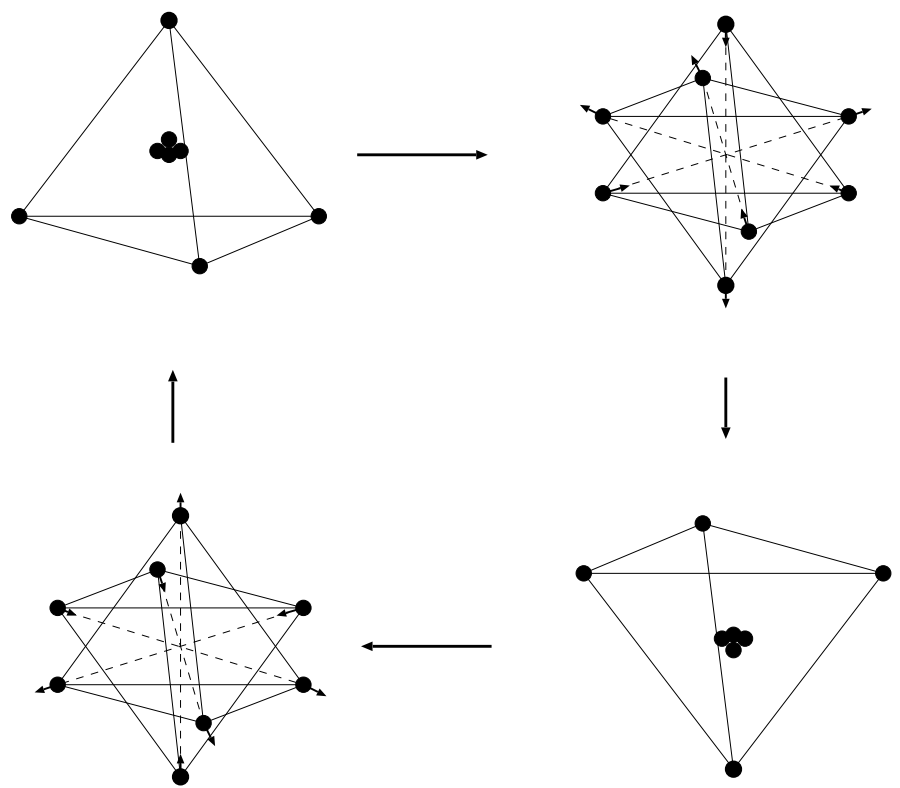

Figure 13: Eight-body orbit whose configuration consists of two dual regular tetrahedrons

In last two examples (4) and (5), we have not checked the assumptions of Theorem 1 nor 2 since the computation is too complicated. But we can undoubtedly expect the existence of orbits.

acknowledgements

The author was partially supported by the Global COE Program "Fostering Top Leaders in Mathematics - Broadening the Core and Exploring New Ground" from the Ministry of Education, Culture, Sports, Science and Technology of Japan.

\section{References}

[1] Broucke, R.: On the isosceles triangle configuration in the planar general three-body problem, Astron. Astrophys. 73, 303-313 (1979)

[2] Chen, K.-C.: Binary decompositions for planar N-body problems and symmetric periodic solutions. Arch. Rational Mech. Anal. 170, 247-276 (2003)

[3] Chenciner, A., Montgomery, R.: A remarkable periodic solution of the threebody problem in the case of equal masses. Ann. of Math. 152, 881-901 (2000) 
[4] Ferrario, D. L., Terracini, S.: On the existence of collisionless equivariant minimizers for the classical $n$-body problem. Invent. math. 155, 305-362 (2004)

[5] Moeckel, R.: A topological existence proof for the Schubart orbits in the collinear three-body problem. Disc. Cont. Dyn. Syst. B 10, 609-620 (2008)

[6] Ouyang, T., Simmons, S. C., Yan, D.: Periodic solutions with singularities in two dimensions in the n-body problem, preprint (available at http://arxiv.org/abs/0811.0227)

[7] Palais, R. S.: The principle of symmetric criticality. Commun. Math. Phys. 69, 19-30 (1979)

[8] Schubart, J.: Numerische Aufsuchung periodischer Lösungen im Dreikörperproblem. Astr. Nachr. 283, 17-22 (1956)

[9] Sekiguchi, M., Tanikawa, K.: On the symmetric collinear four-body problem. Publ. Astron. Soc. Jpn. 56, 235-251(2004)

[10] Shibayama, M.: Multiple symmetric periodic solutions to the 2n-body problem with equal masses. Nonlinearity 19, 2441-2453 (2006)

[11] Venturelli, A.: A variational proof of the existence of von Schubart's orbit. Disc. Cont. Dyn. Syst. B 10, 699-717 (2008) 